\title{
Variables Influencing RNAV STAR Adherence
}

Michael Stewart, Bryan Matthews, Vijay Janakiraman, Ilya Avrekh

In this study we investigated how variables in the aviation domain impact adherence levels of aircraft flying area navigation arrivals with optimized profile descents (RNAV OPDs). Variables categories were: weather, aircraft, procedure, and traffic. Non-adherence events analyzed were: miss above, miss below, skip before merge, and skip after merge. Miss below and miss above describe when a flight does not comply vertically with a procedure. Skips refer to a flight leaving a procedure, then returning. Findings of this work reveal that vertical events are most impacted by altitude restriction size, steepness of flight paths, and merging routes. Lateral events were impacted by merging flight conflicts, number of speed restrictions, and the flow rate of the arrival traffic. This study helps increase understanding of how the system is functioning and identifies where procedures are not flexible enough to handle the variability in normal operations. major airports, procedure design, and recommendations for future work. 


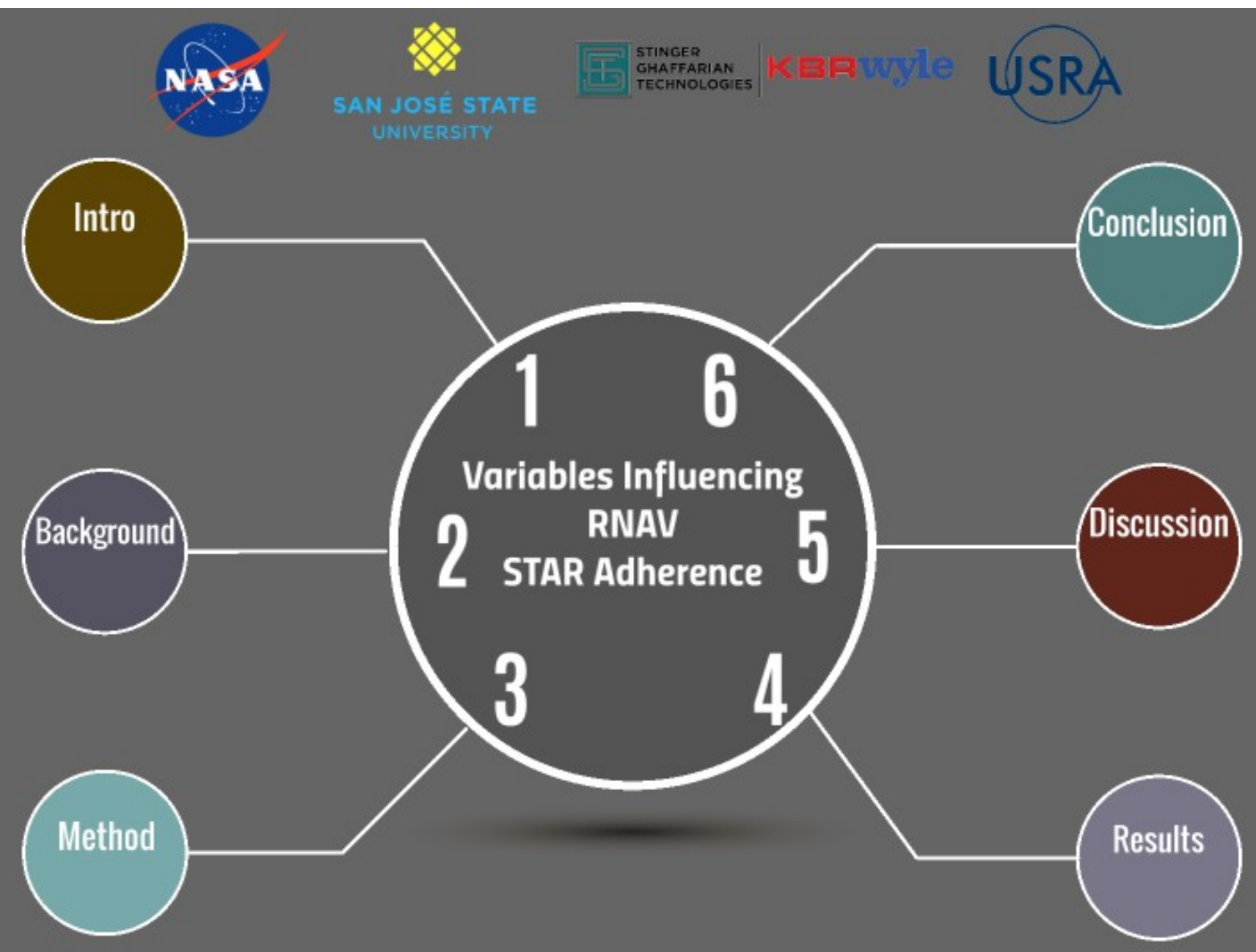




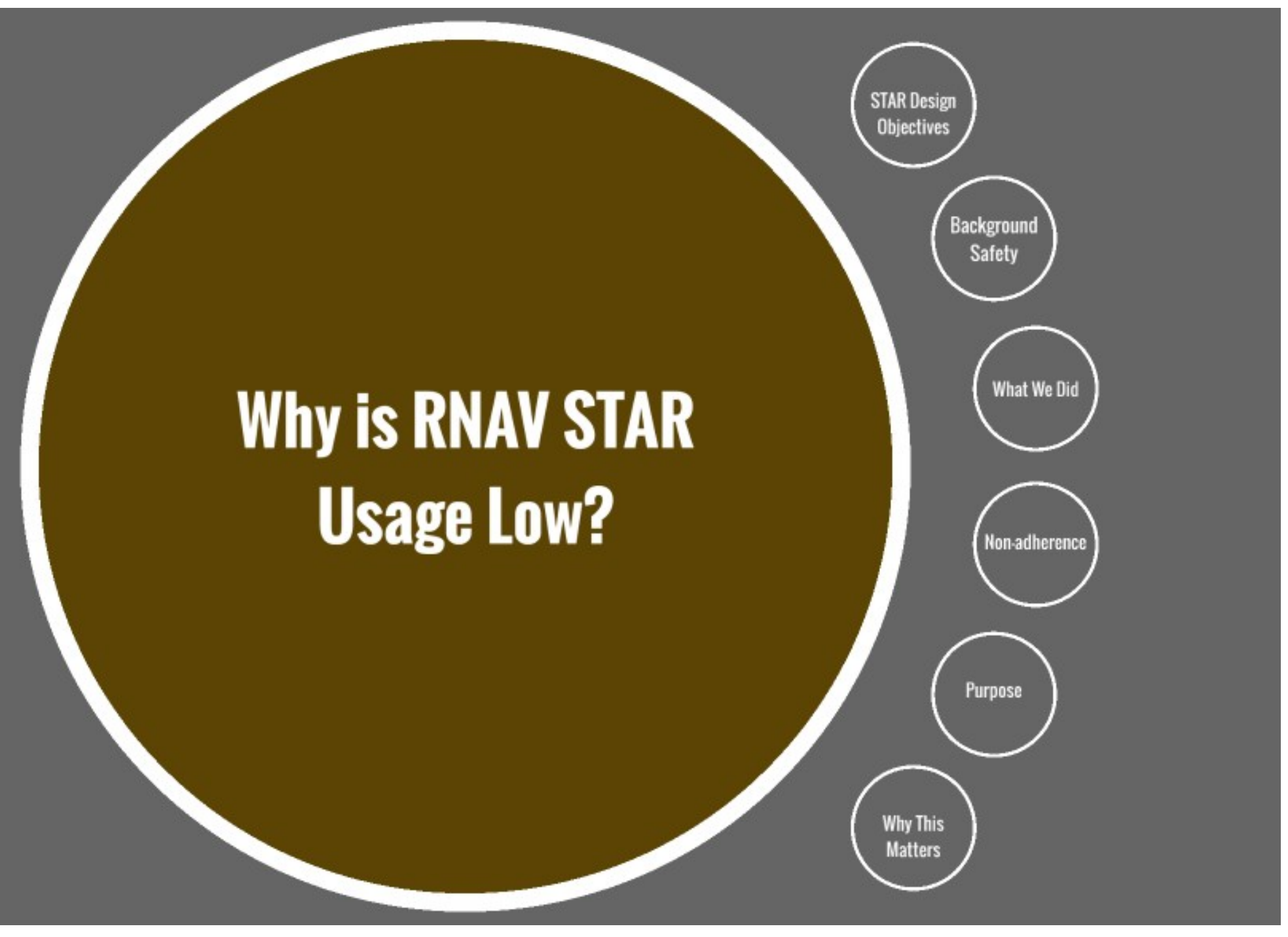




\section{STAR Design Objectives}

Fuel savings from constant descent

Noise reduction

Predictable flight paths

Reduced Communication

NOTE: Descond vio Mach number unil tronsation b 290

NOTE: Rodor roquirod. 旅

NOTE: DME/DME/IRU or GPS required. NOTE: Turbojet dirrrof only. CASOT
$240 \overline{290 \mathrm{~K}}$

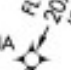

BOWUNG GREEN OE AXXEL
FL240 $290 X$ BWG

NOTE: Turbojet oircroft only.
NOEqired.




\section{Safety lssues}

\section{Commercial Aviation Safety Team (CAST) safety enhancements (SE)}

\section{SE 213 Addresses pilot and controller}

procedures, and charting depiction for the purpose of error reduction.

SE 214 Addresses path compliance

issues related to procedure design.

Subjective reporting (e.g., ASRS, ATSAP, ASAP) was used as the dependent variable in this work. These reports may not be representative of

occurrence rates or category saturation. Quantitative metrics are needed. 


\section{What we did}

Measured non-adherence to STARs by integrating data. We used radar for position and compared them to the three dimensional 3D paths.

\section{Data Types:}

Procedure, weather, traffic, and aircraft (outside user control) 


\section{Operational Definition of non-adherence events}
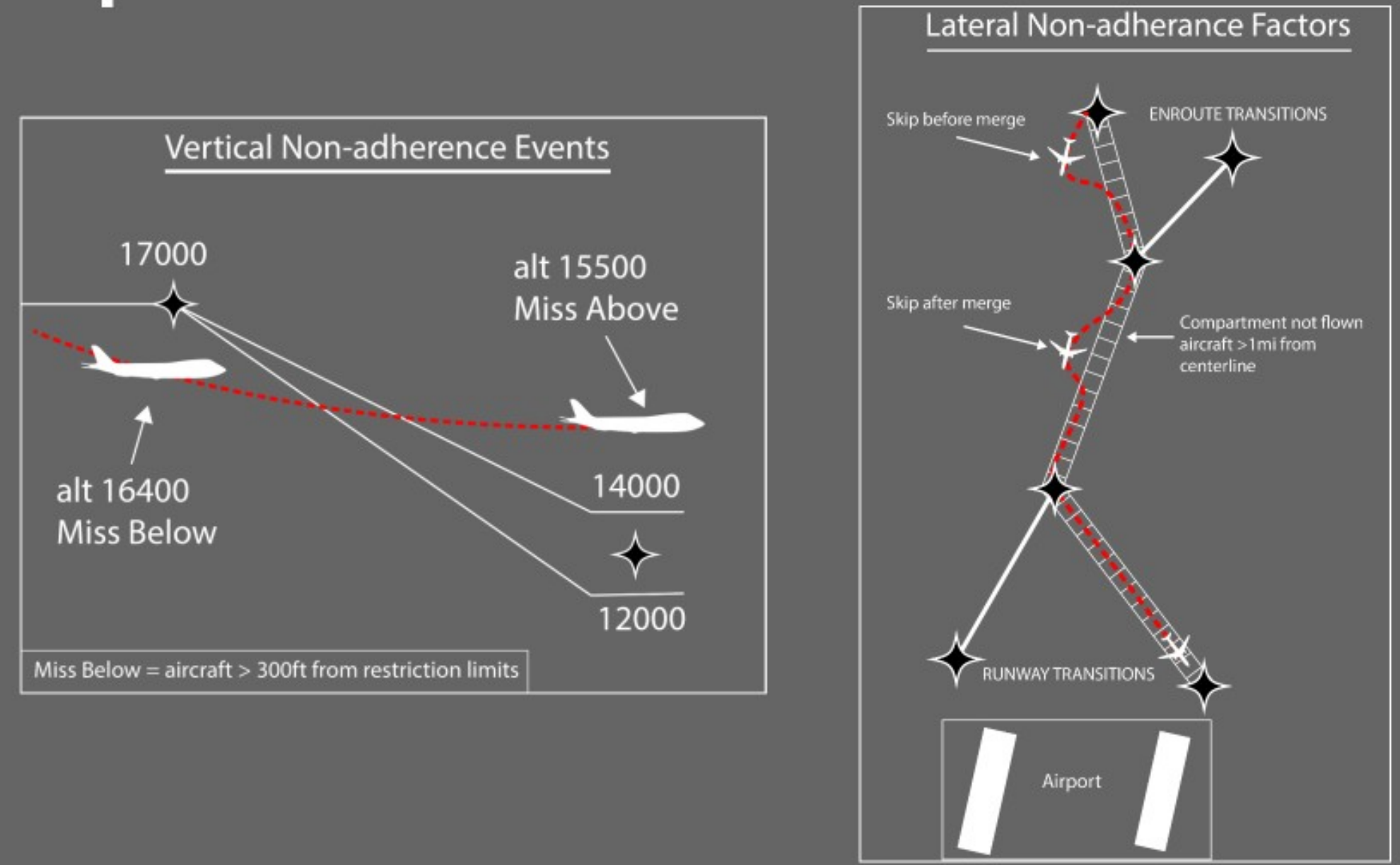


\section{Purpose}

1. To create a measurement system for adherence and terminology to describe it.

2. To see if variables outside of the users' control are predictive of adherence to the procedures. 


\section{Why this matters}

The system is handling flights, but we have no way to determine if it's because of the design or in spite of it. 


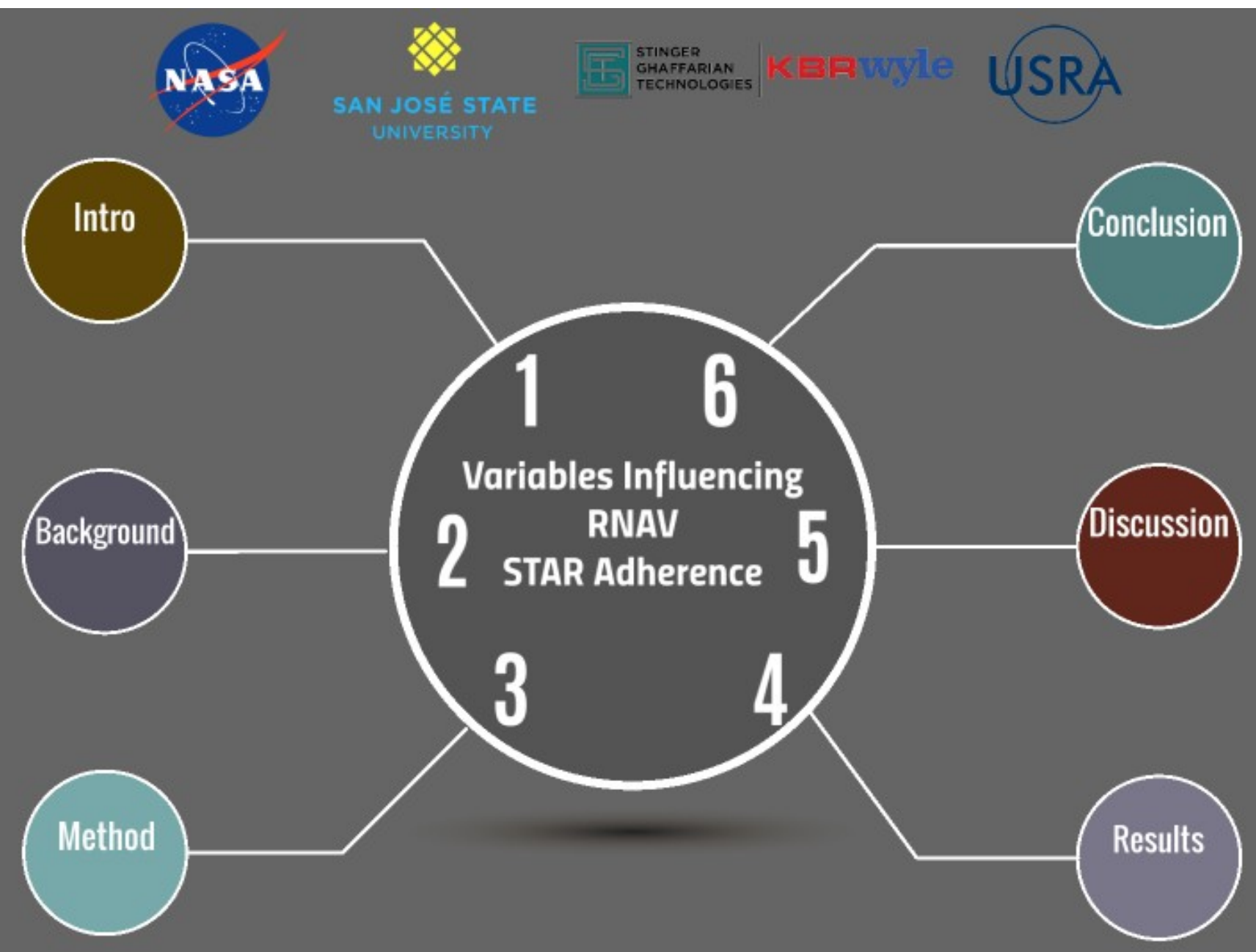




\section{Background}

Measurement

Human

Performance

Issues

What's been done and how this study fits

RADI

Variables 


\section{Performance Variability \& Measuring Adherence}

Johnson, 2009 Performed a human-in-the loop simulation to identify possible variables to impede STAR usage. They found aircraft weight, type, wind,

Limitations: simulation is limited in scope and validity.

Stewart \& Matthews, 2017 measured adherence of 24 major US airports. Median full adherence ranged from $0 \%-24 \%$.

Limitations: measurement granularity, radar sampling rate, and transition assignment. 


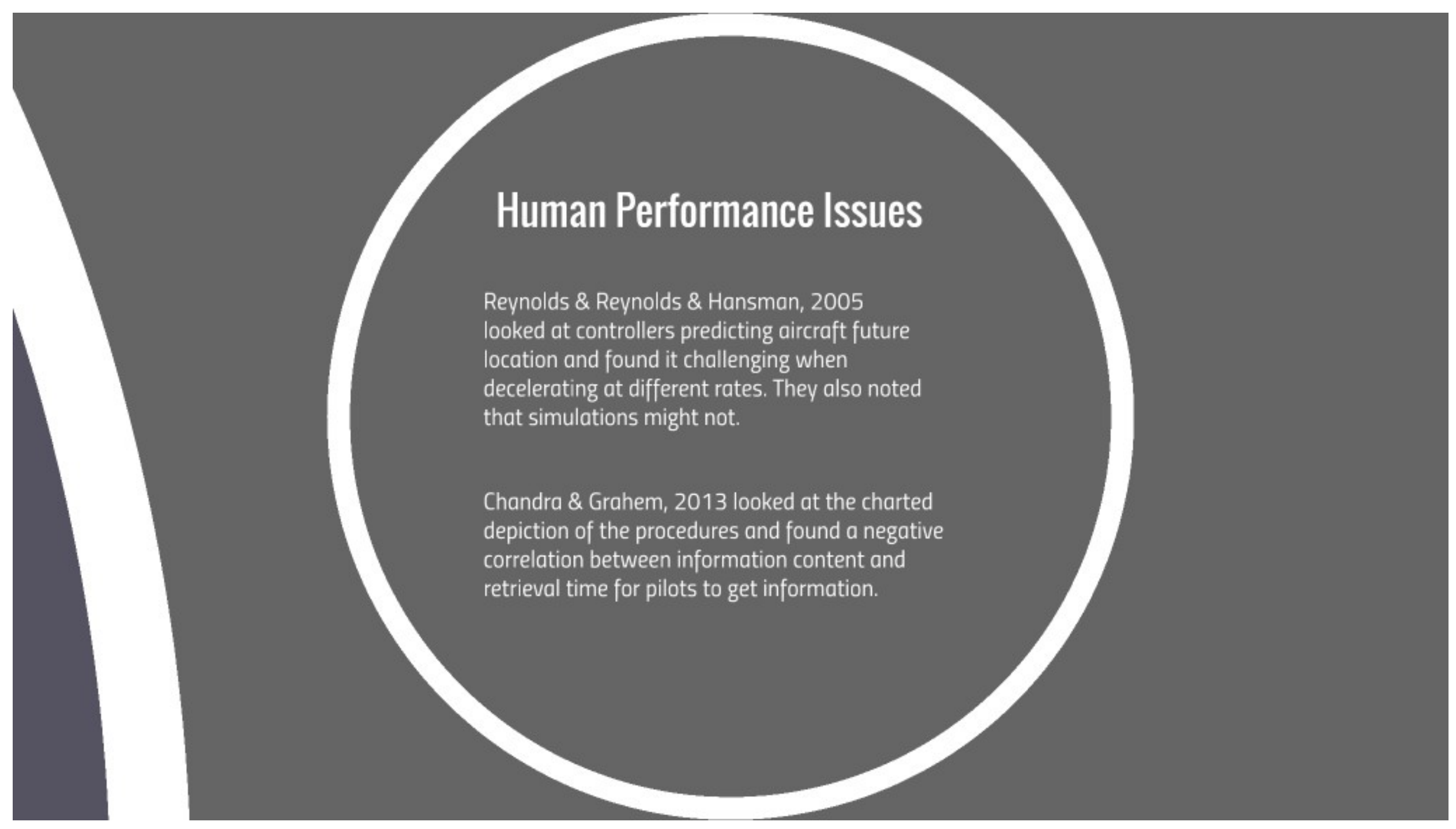




\section{RNAV Adherence Data Integration}

This system gives us a monitoring capability to learn how our system is functioning.

RADI Data Processing Pipeline

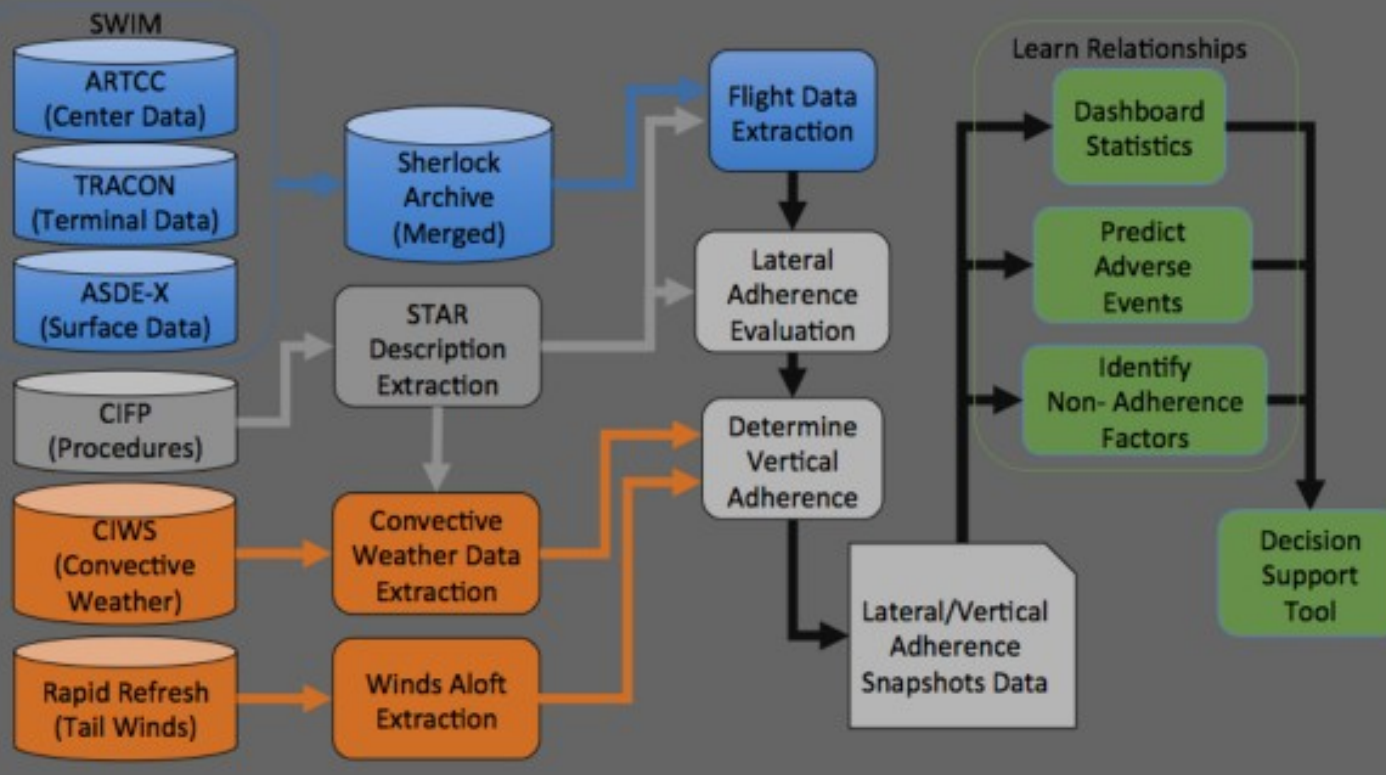




\section{Variables}

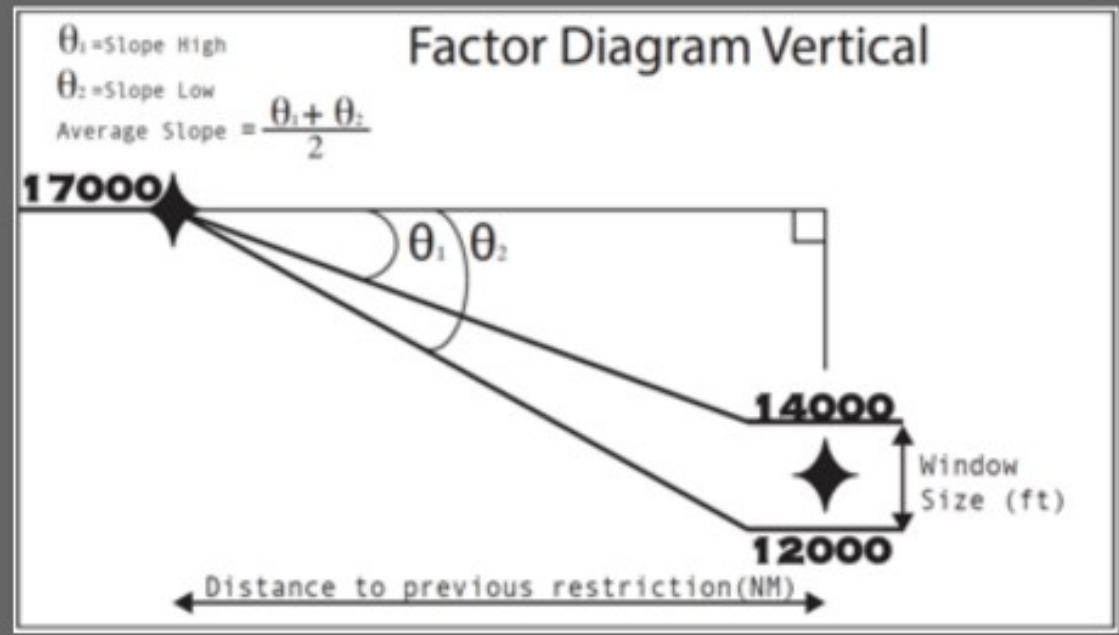

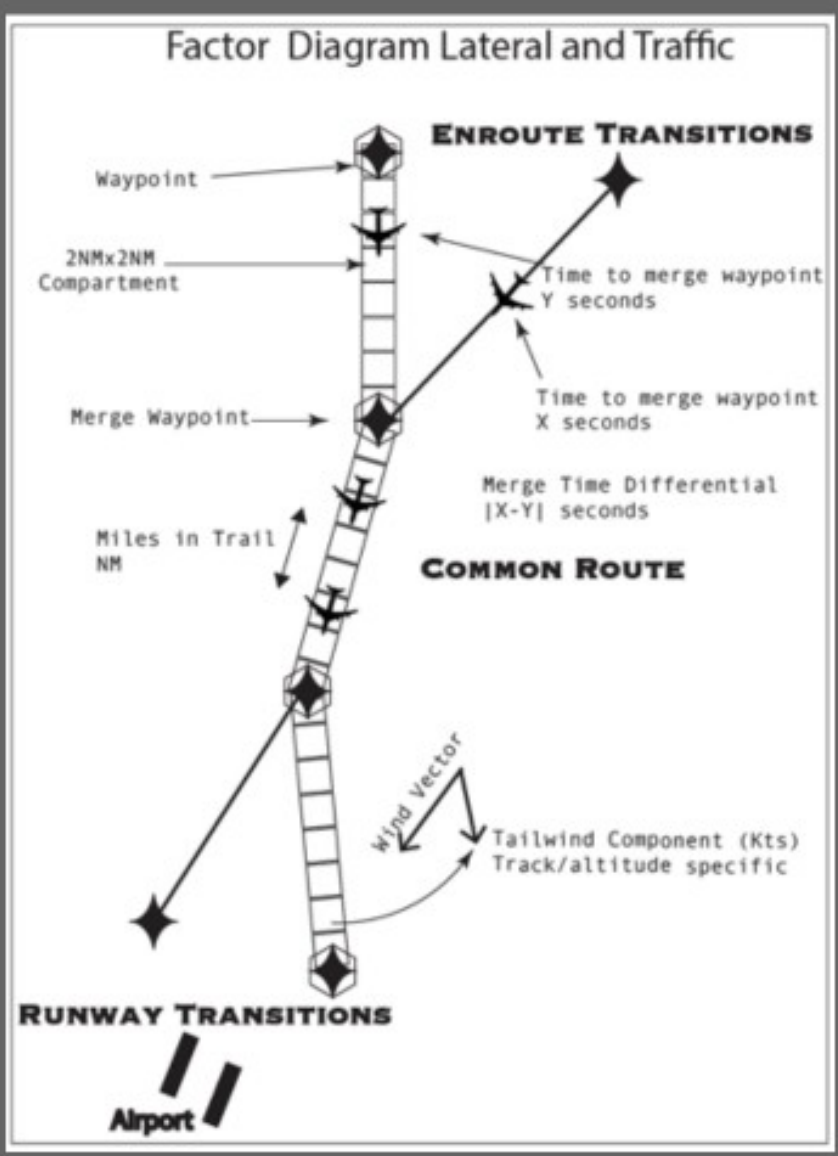




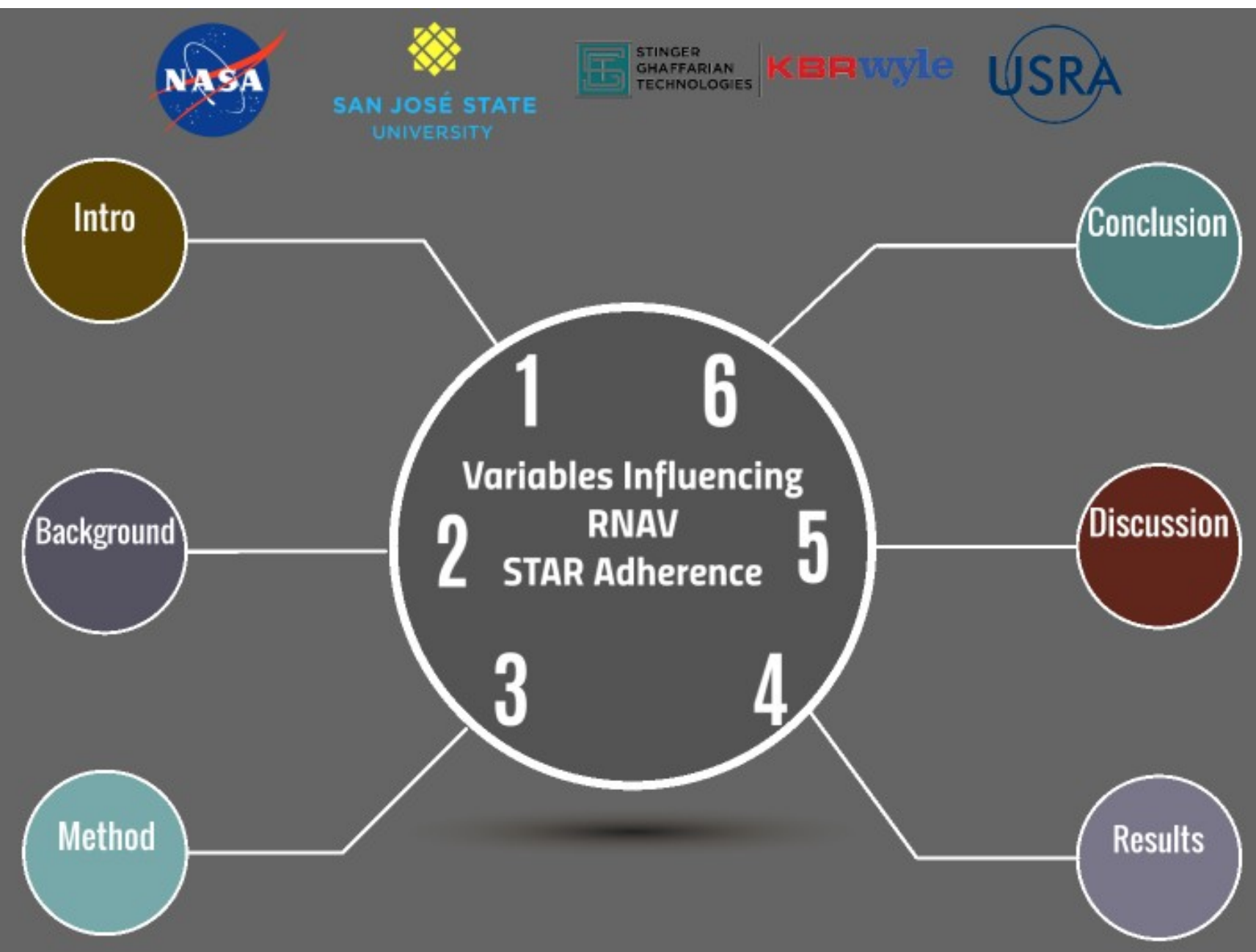




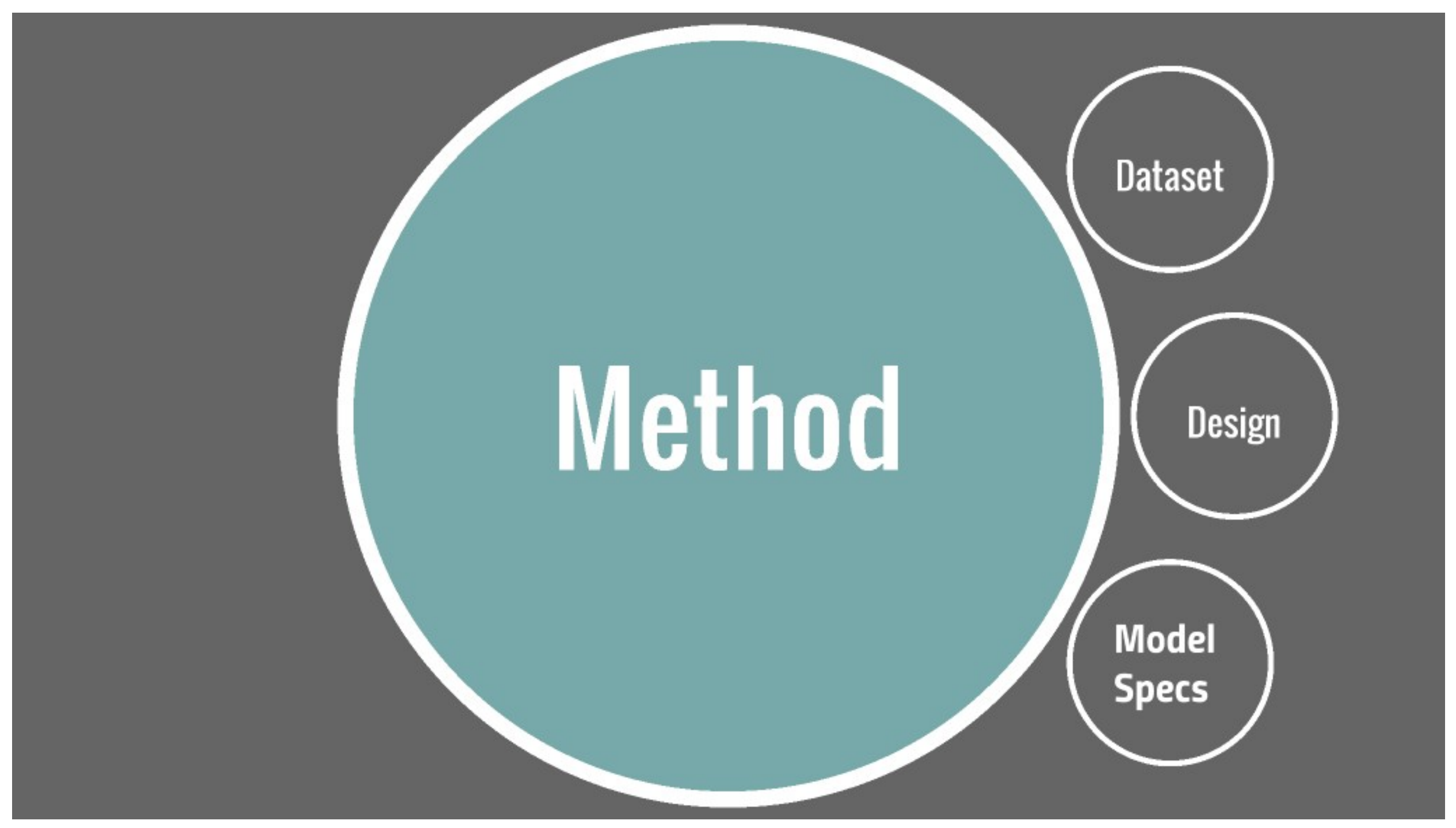




\section{Dataset}

32 Major US Airports 276 RNAV STARs

$12,762,967$ flights

of these

10,619,068 Flew some portion of a STAR

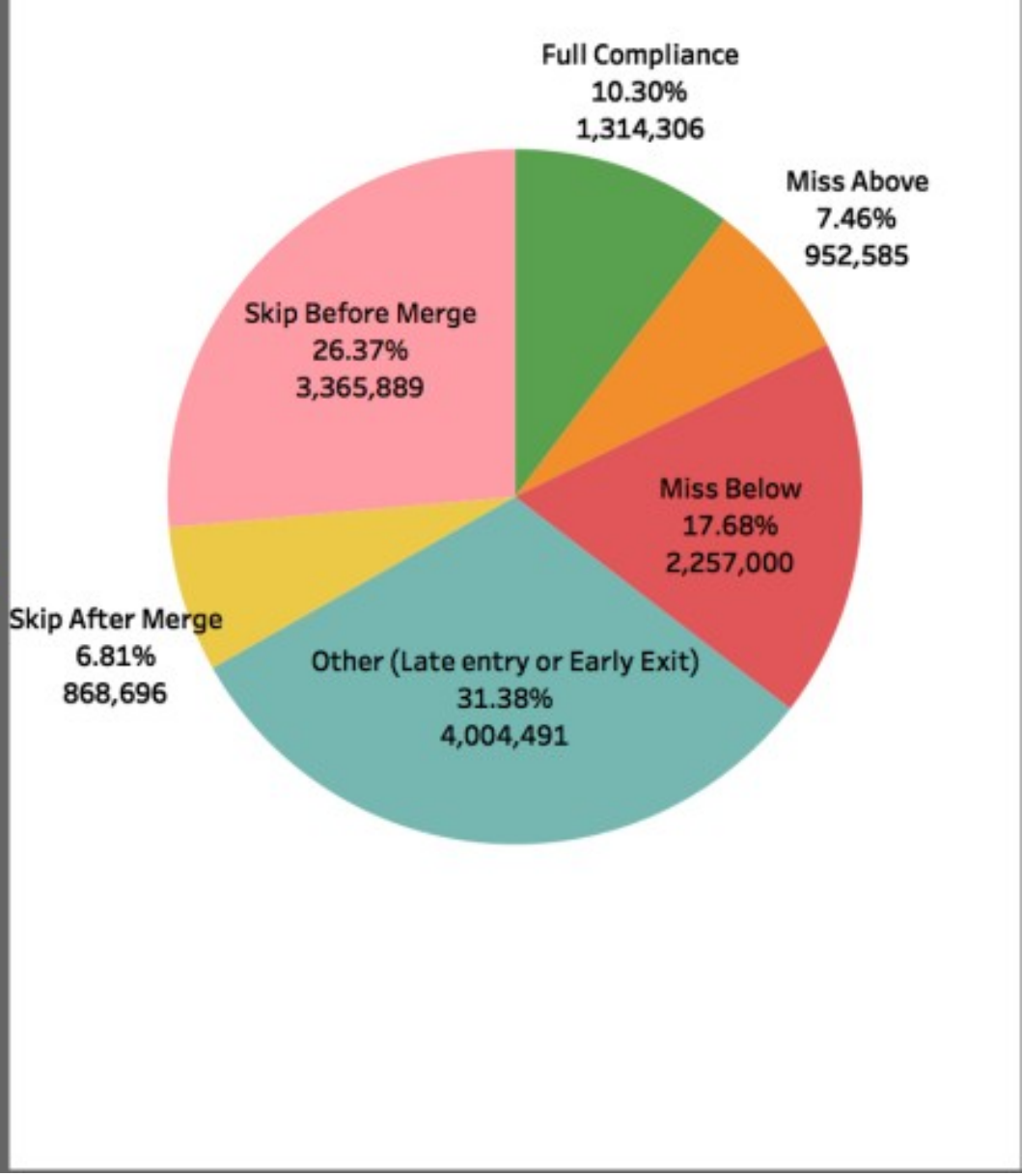




\section{Design}

Supervised predicative machine learning using classification decision trees

4 Dichotomous variables (events):

- miss above

- miss below

- skip before merge

- skip after merge
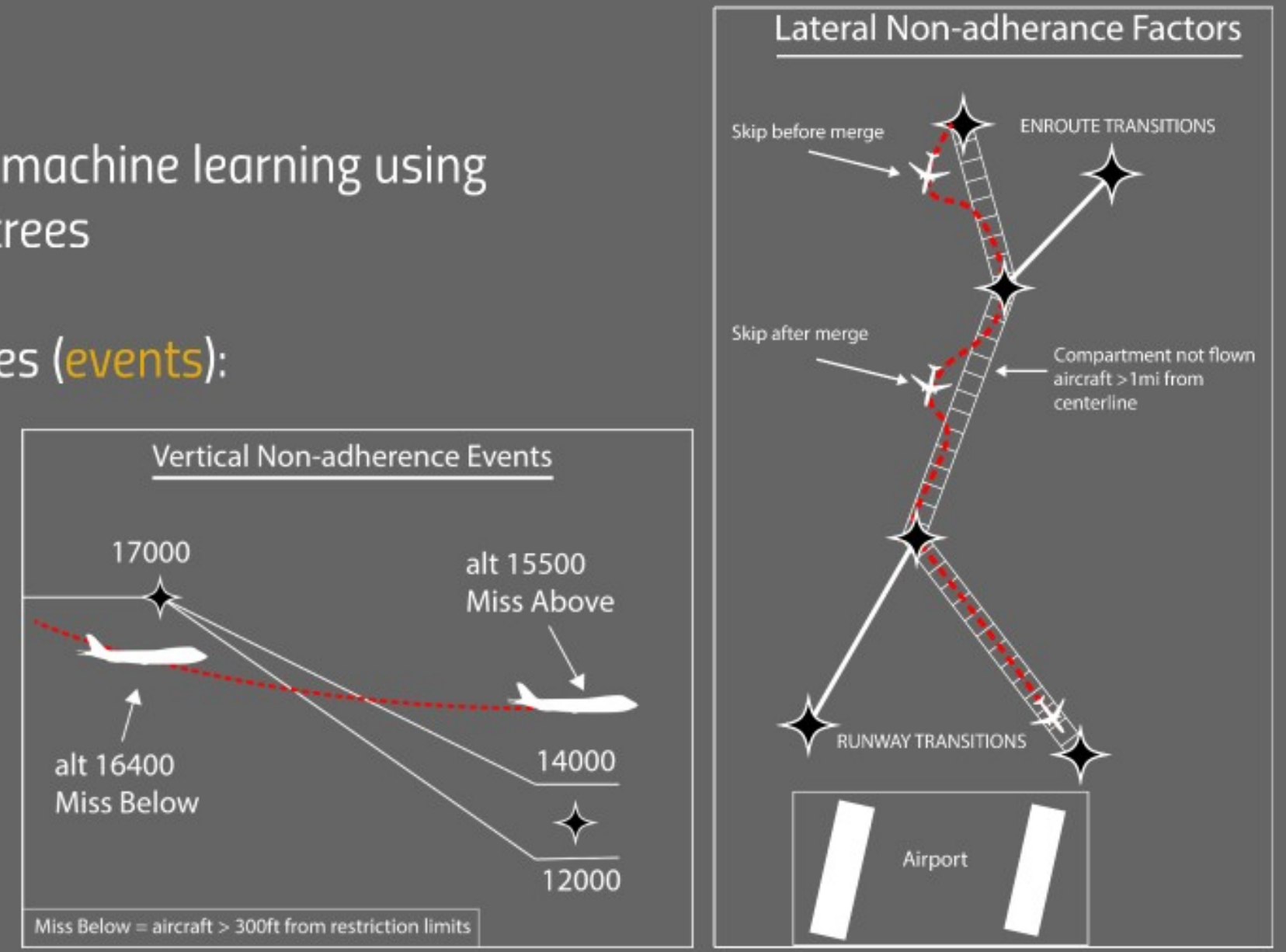


\section{Model Specs}

Miss above/below

Average slope, distance to previous restriction, altitude window size, tailwind, merge waypoint type, and waypoint percentage

Skip before merge \& Skip after merge

Merge time differential, Tailwind differential, STAR flow, Number of speed restrictions, Miles in trail, and Weather on compartment, regional flight, and waypoint Percent 


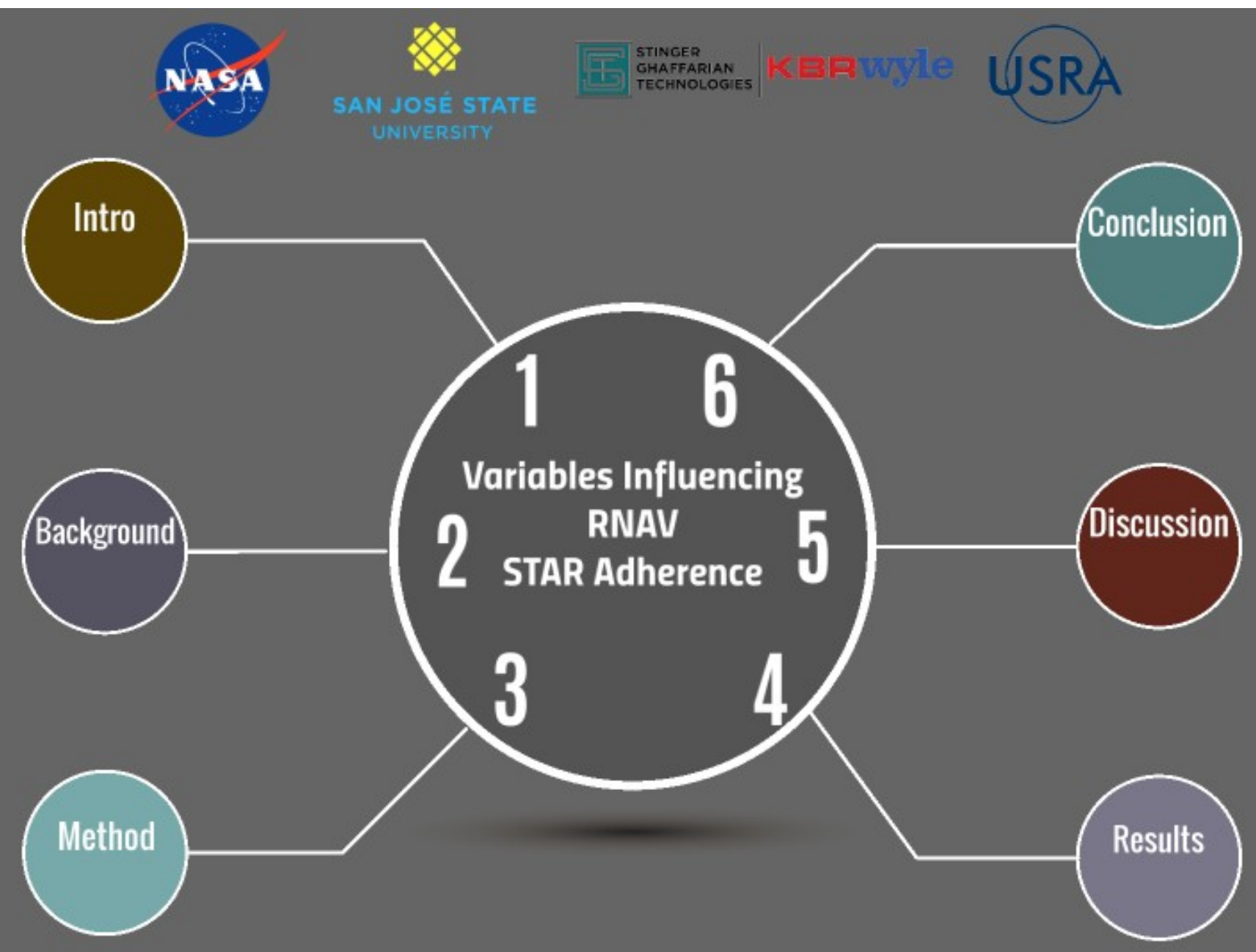




\section{Results}

Skip after merge did not meet $60 \%$ and was excluded.

Skip before merge adherence did not meet $60 \%$ and was excluded.

Miss Above
$70 \%$ Accurac
$65 \%$ TP
$74 \%$ TN

Miss Above
$70 \%$ Accuracy
$65 \%$ TP
$74 \%$ TN

Miss Above
$70 \%$ Accuracy
$65 \%$ TP
$74 \%$ TN

Miss Above
$70 \%$ Accuracy
$65 \%$ TP
$74 \%$ TN

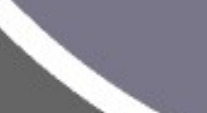

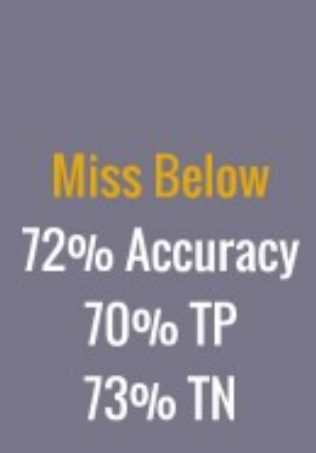

Skip Before

Merge

60\% Accuracy

$62 \%$ TP

$\%$ TN 


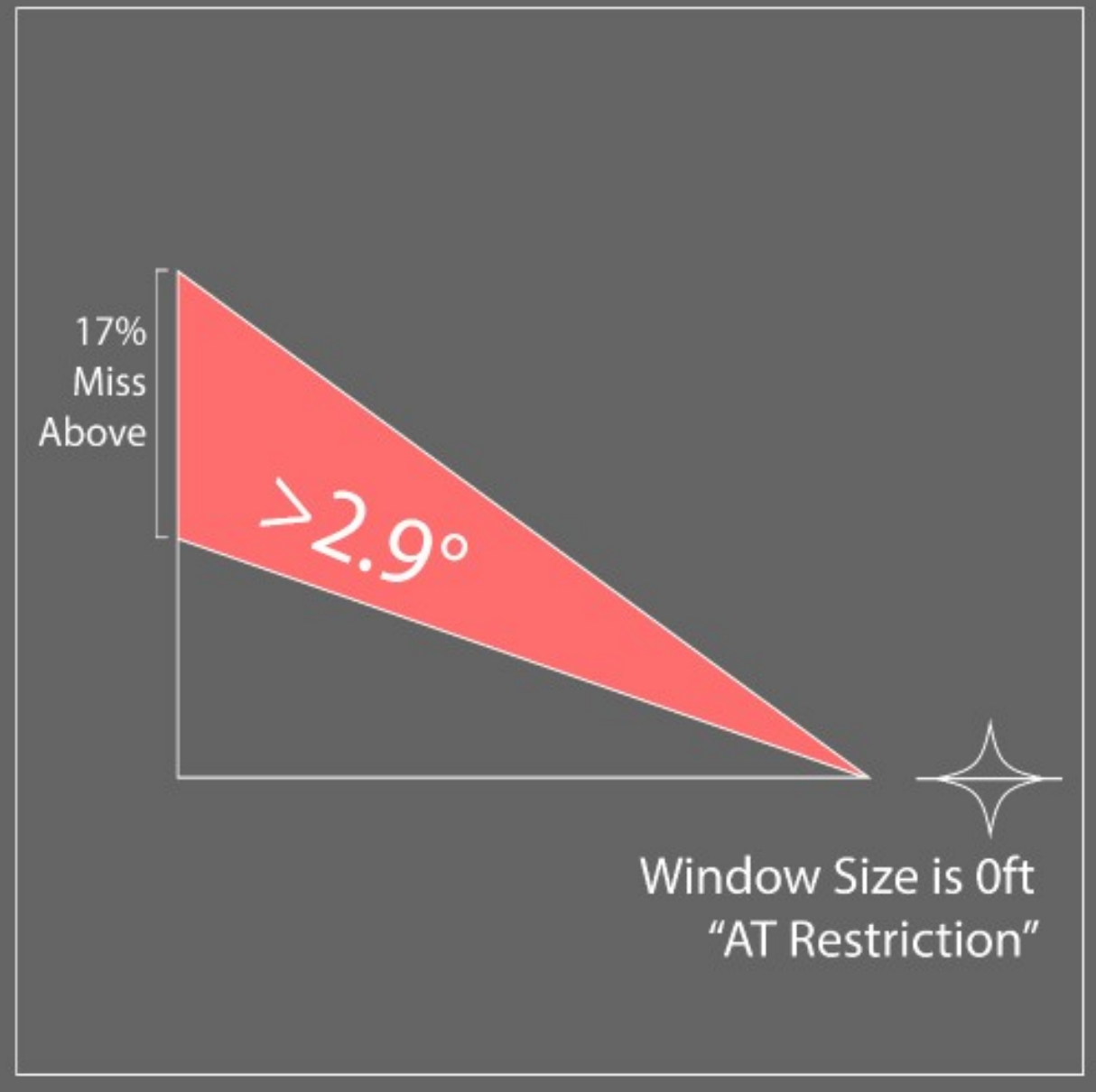




\section{Adherence}

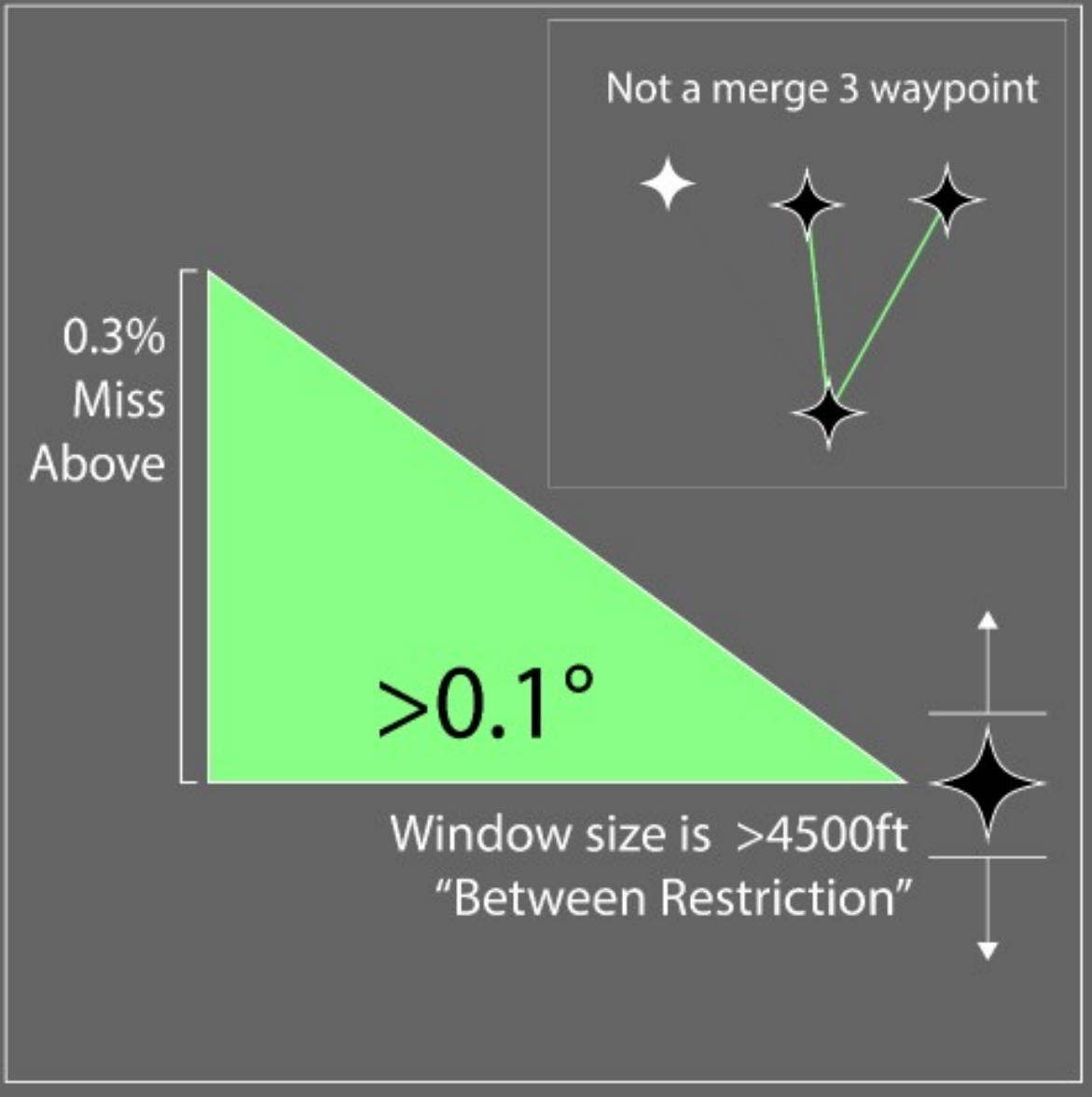




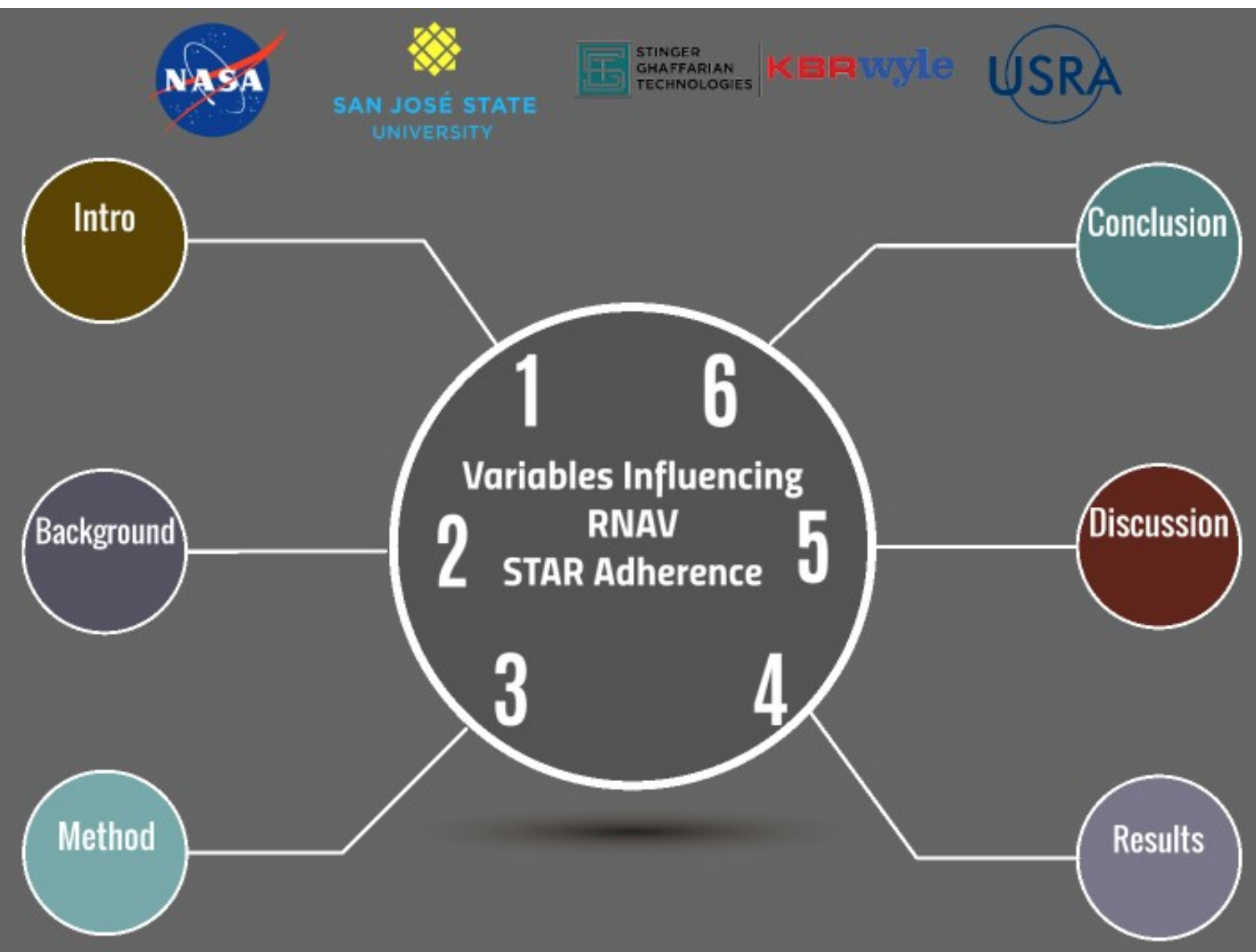




\section{Discussion}

Variables predict about $70 \%$ for vertical and $60 \%$ for lateral events

Interactions matter

Merging paths predicted non-adherence both vertically and laterally.
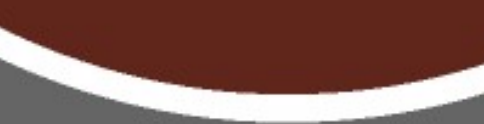


\section{Unexpected Outcomes}

Convective weather wasn't as impactful as we thought. (could be measurement, sparsity, or a combination).

Lateral non-adherence decreased as speed restrictions increased. (possibly from increased control of traffic the traffic flow or that controllers choose to use them differently). 


\section{Practical Applications}

Using rules to look for problems or the absence of problems

24 active waypoints have these

characteristics

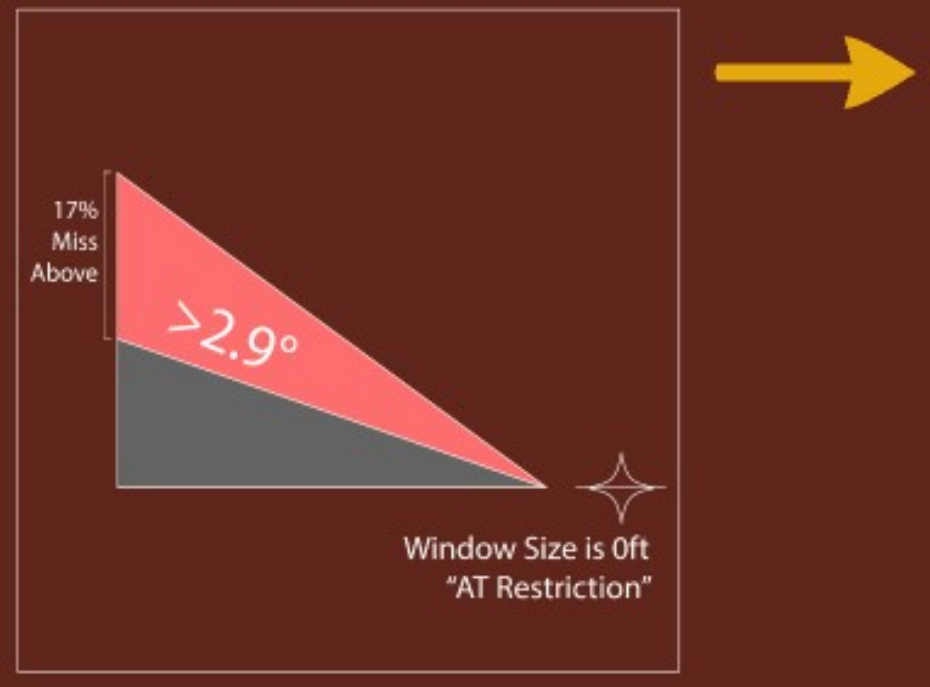

\section{ASRS Reports}

Waypoint: LIQWD

"The Captain stated we could not make the next crossing altitude restriction for LIQWD intersection at 6,000 feet. I called out our airspeed at 224 knots to when I noticed us speeding up passed our assigned 210 knots.

\section{Waypoint: ARRTU}

"Last week, I witnessed the airplane in VNAV PATH unable to comply with published speed as it descended via the arrival between HEFLY and ARRTU. It had difficulty because the airplane inexplicably chose to cross HEFLY at the FL190 high end of the published altitude and thus had virtually no chance in crossing ARRTU at 10000 and 250 kts."

\section{Waypoint: COPEN}

"From a pilot's point of view this arrival could be better, safer, and more user friendly by simply extending the distance from BLUZZ and COPEN intersection. 9.7 miles is simply not enough to lose altitude and slow down. Could it be increased to possibly 20 or 25 miles? The chart states that from COPEN intersection to the airport is $40 \mathrm{NM}$, surely there is enough room to make this possible.

\section{Waypoint: COPEN}

"I briefed that I follow the VPI (Vertical Path Indicator) as habit on ODP (Optimized Descent Profile) arrivals, and waited until the VPI carrot came alive to begin descent. While we met BLUZZ below 23000, as soon as we crossed the fix the FMS messaged "max grade unable next altitude." We advised ATC we were unable to cross the COPEN fix at 10000. ATC handed us off to approach, who gave us a vector to join the approach to $18 \mathrm{~L}$. 


\section{Limitations}

Not causal

No intent information

No airspeed information

More information is still needed to predict performance 


\section{Next Steps}

Transition to aircraft sensor (FOQA) data instead of radar for increased information.

Perform observational research of ATC and flight deck personnel to get a better understanding of causal issues. 


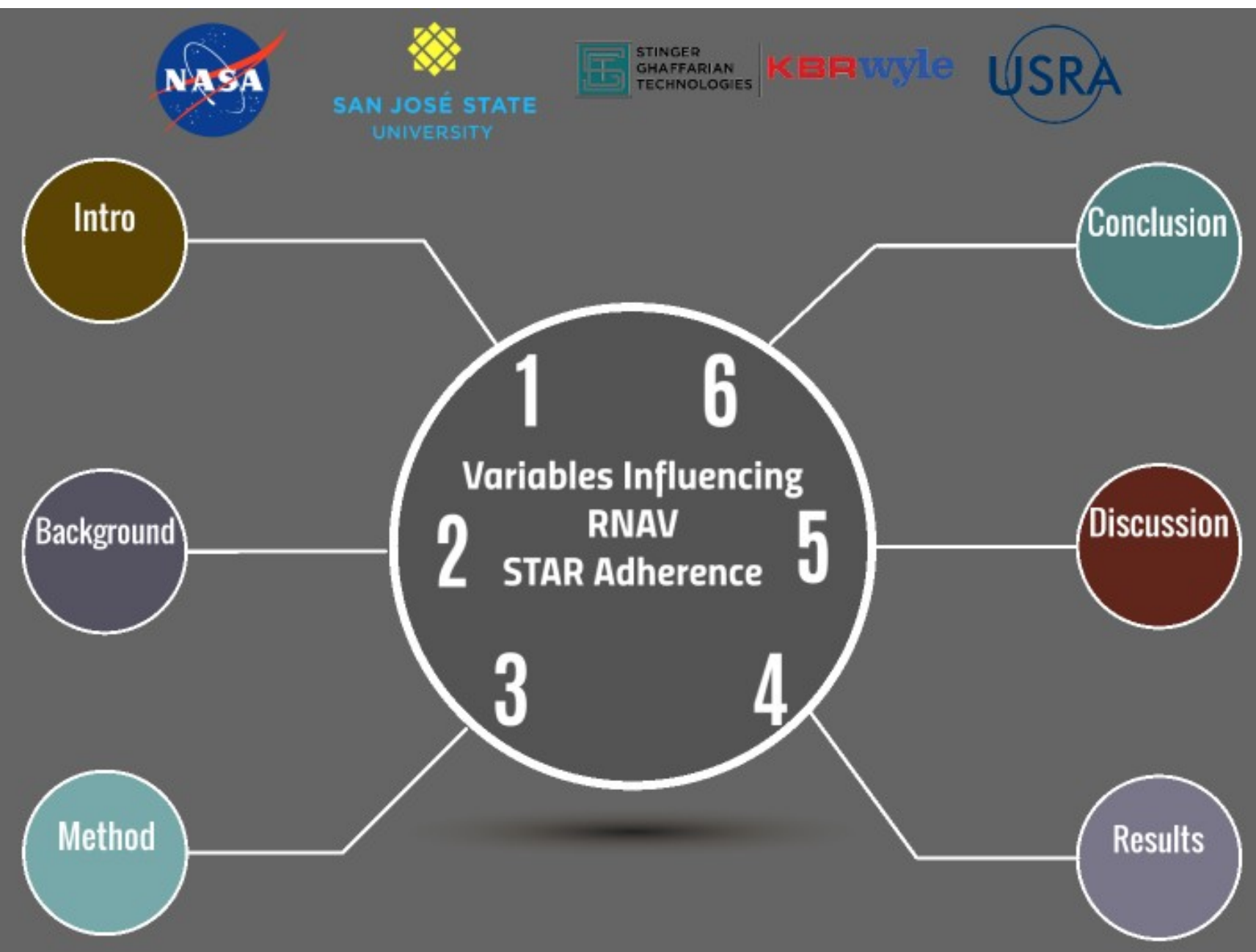




\section{Conclusion}

Non-adherence shows us that the domain is too dynamic to be handled by a static procedure.

We need to understand how/why controllers and pilots are adapting to enhance the system's performance.

Monitoring procedure performance gives us the ability to learn and adapt.

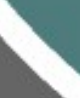

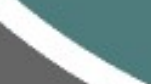




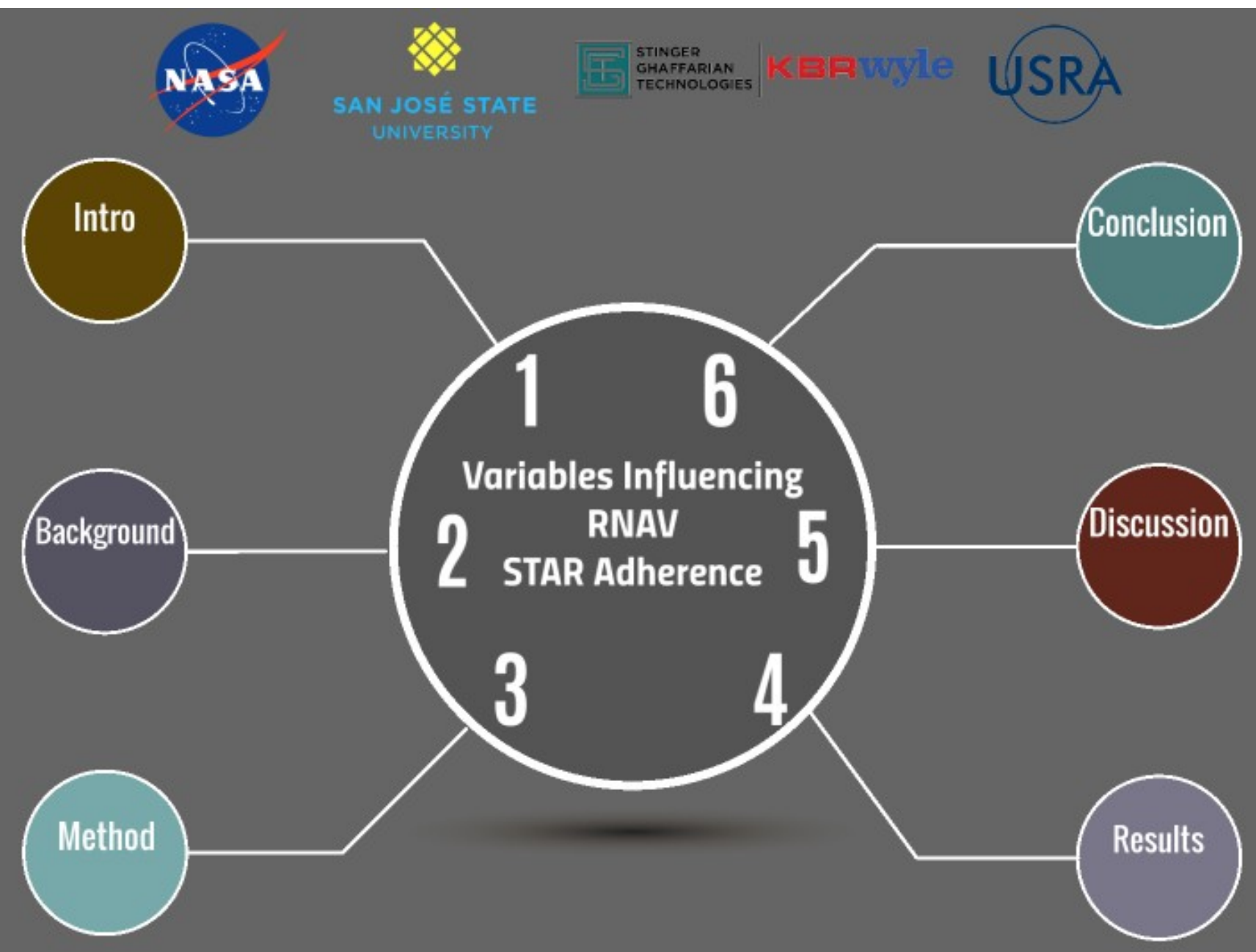

